\title{
Interfacing and Controlling of Liquid Crystal Display with Single Board Computer for Communication Access Terminal
}

\author{
G.Hemavathi $^{1}$, J.T.Pramod ${ }^{2}$ \\ M.Tech Student, Dept of Electronics and Communication Engg, Madanapalle Institute of Technology \& Science, \\ Madanapalle, A.P, India ${ }^{1}$ \\ Asst Professor, Dept of Electronics and Communication Engg, Madanapalle Institute of Technology \& Science, \\ Madanapalle, A.P, India ${ }^{2}$
}

\begin{abstract}
Advanced Composite Communication System is an IP based integrated communication system designed to provide quick and reliable communication over Very Low Frequency, Medium Frequency, High Frequency and Very/Ultra High Frequency bands on board naval ships and submarines for facilitating ship to ship, ship to shore and ship to air communication. The Communication Access Terminal (CAT) is an important and integral part of ship communication system. CAT is a PC104 Single Board Computer (SBC) based system for internal and external communication. To provide visual information to the end user about line status, network status, information about connected radio number, CAT number etc. As a part of CAT development, interfacing of LCD board to PC104 Mother Board using RS232 based serial protocol is the main objective of this project. Programming the board is done using Qt Creator in C++ language. The Liquid Crystal Display interfaced to the PC104 mother board is tested using Graphical User Interface (GUI) application developed in Qt. The testing steps of display include 1) Displaying a preloaded logo on startup. 2) Faulty display detection using full pixel illumination. The current CAT is a simplified version of earlier CAT, which best suits for extreme weather conditions. The current CAT is designed so as to withstand the temperature range of $-40^{\circ} \mathrm{C}$ to $+85^{\circ} \mathrm{C}$, relative humidity up to $80 \%$ at $25^{\circ} \mathrm{C}$, vibration $10 \mathrm{~g}$.
\end{abstract}

Keywords: PC104 Mother Board, Communication Access Terminal, RS232 serial protocol, Qt creator, Graphical User Interface, Liquid Crystal Display.

\section{INTRODUCTION}

Now a day's embedded systems are playing a vital role in our surrounding areas such as electronic calculators, home appliances, printers, scanners, digital cameras, displays and many more. In many of the embedded system applications programmable boards are developed to do specific tasks according to the user requirements. Modern embedded systems are often based on micro-controllers but ordinary microprocessors are also still common, especially in more complex systems.

Displaying data on different displays is also one of the embedded system applications.

We have many different types of displays such as seven segment displays, LCD etc. To show text on display we should give control commands to display through any single board containing microcontroller or microprocessor by interfacing LCD to the Single Board Computer. In the same way, to display the required information on LCD one single board computer has chosen. In order to do that Liquid Crystal Display is interfaced to PC104 Single board computer to display text related to Communication Access Terminal.

Communication Access Terminal is one of the sub-parts of Advanced Composite Communication System.

\section{HARDWARE AND SOFTWARE REQUIREMENTS}

\section{A. A. Hardware Requirements:}

This project requires some hardware components such as PC104 Mother board, KIB380 interface board, Compact flash card, LCD, Power supply.

\section{a) PC104 Mother Board:}

The development of this project uses PC104 Mother Board (CPC30401 version). Pre-installed operating system supplied for PC104 is FDOS 6.22 operating system and also it supports MSDOS, windows CE 5.0, CE 6.0, XPe, as well as QNX 6.3X and Linux 2.6 operating systems. This board is designed for low power consumption wide operating temperature range. All basic components like memory, chipset, periphery controllers and CPU are soldered on board providing very less vibration and of protective conformal coating. Wide variety of data display capabilities are offered by this board provided industrial operating temperature range of this board is $-40^{\circ} \mathrm{C}$ to $+85^{\circ} \mathrm{C}$. The available memory, ports etc. are shown in below figures:

In this figure if we observe, we can find number of jacks for different connections, LED's, Watch Dog Timer, Reset button, PCI \& ISA slots, CPU, BIOS, PCI to ISA bridge, CPU and Buzzer etc. 


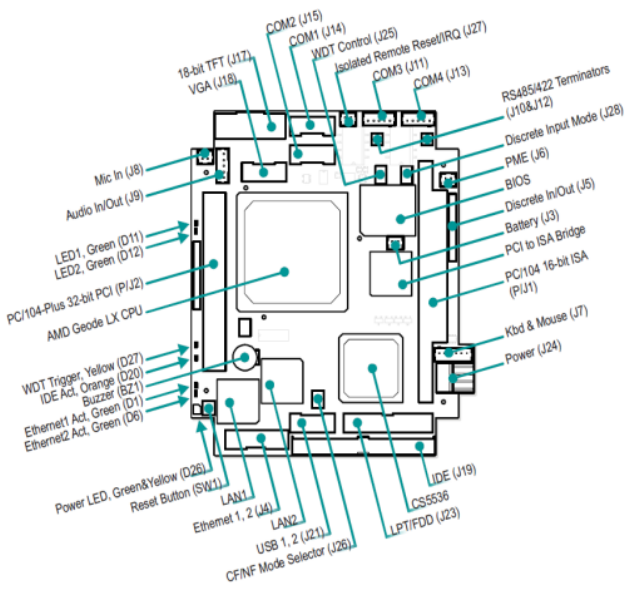

Top view of PC104 board

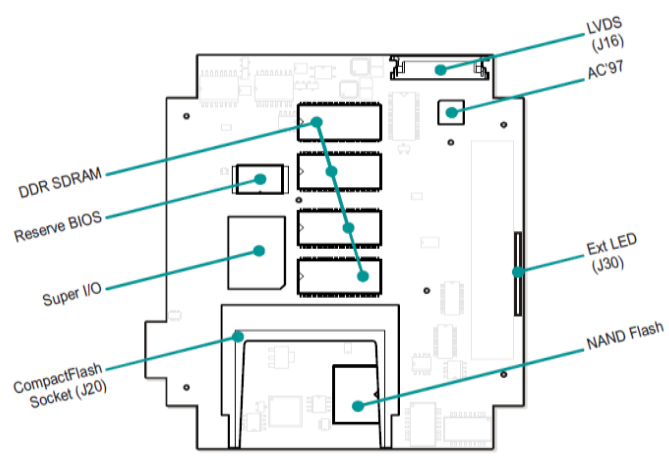

Bottom view of PC104 board

This board also contains $1 \mathrm{~GB}$ of on-board NAND flash, $256 \mathrm{MB}$ of RAM and Compact flash socket to insert the compact flash card at the bottom side of the board.

This is the schematic view of PC104 board. The above figures are top and bottom views. In top we can see onboard chips, jacks for different connections etc. In the bottom view we have DDR

SDRAM of size $256 \mathrm{MB}$, reserve BIOS, compact Flash Socket, NAND Flash etc.

b) KIB380 interface board:

KIB380 makes interface of the processor module available via standard PC connectors-VGA, Audio (Mic, Line In, Line Out), four COM ports, two LAN, two USB, two PS/2 for keyboard and mouse and LPT.

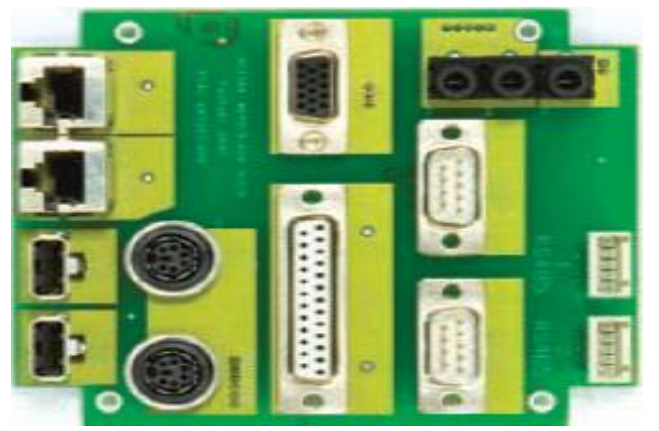

Top view

In this top view we have all ports for connecting external devices like keyboard, mouse, monitor, LAN connections.

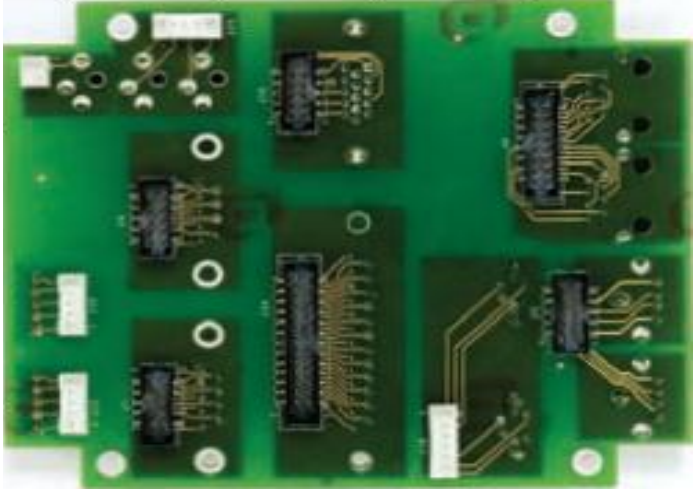

Bottom view

Bottom view of interfacing board will as above. Ribbon cables from jacks of PC104 connected here to the corresponding ports for correct communication.

c) Compact flash card:

This device can be used as a bootable disk or can be disabled in BIOS setup. The capacity of compact flash memory chip is 4GB. We have the option of installing operating system to this bootable disk.

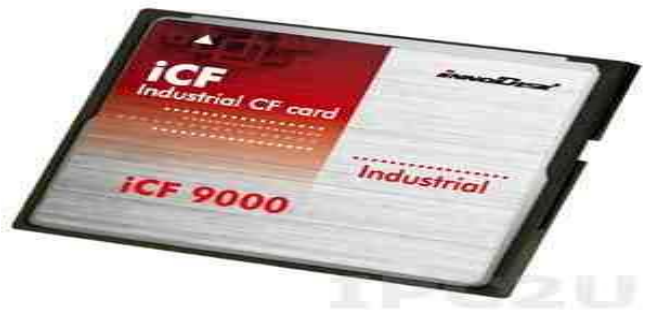

The compact flash memory chip will be look like this. This is an industrial $\mathrm{CF}$ card to operate in wide temperature ranges from $-40^{\circ} \mathrm{C}$ to $+85^{\circ} \mathrm{C}$.

\section{d) Liquid Crystal Display:}

The LCD is an alphanumeric Liquid Crystal Display designed for providing quick solution to any project. For this LCD available protocols are RS232, TTL and $\mathrm{I}^{2} \mathrm{C}$ protocols in the standard model.

Communication speed of this LCD is up to $115.2 \mathrm{kbps}$ for serial protocols and $100 \mathrm{kbps}$ for $\mathrm{I}^{2} \mathrm{C}$ protocol. On board memory for this LCD are 40 custom characters which can be saved and called for bar graphs, startup screens or large numbers. The view of LCD will be as below:

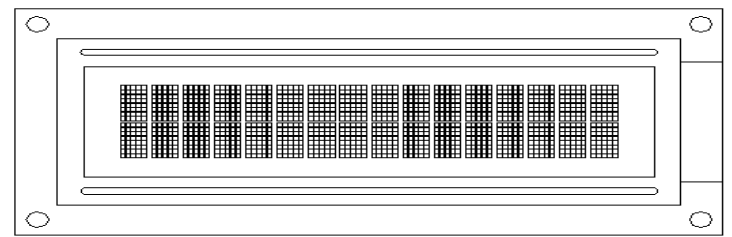

Front view of LCD

By observing the top view of LCD we can find that it is a pixel based display. On this display clarity is more compared to other displays like seven segment display. 


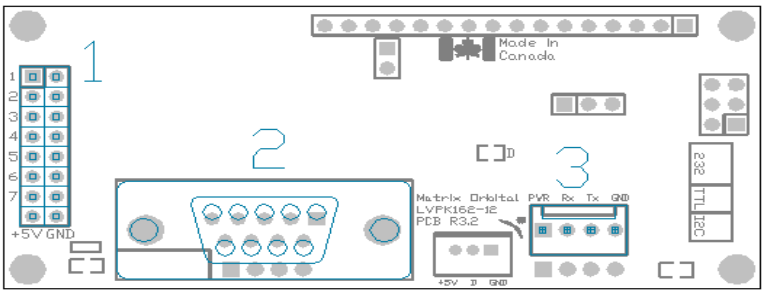

Back view of LCD

This is the bottom view of LCD here used parts are highlighted by giving numbers. 1. General purpose Input/output, 2. Serial DB9 connector,

3. Communication/power Header. In the name itself we can find the working of those connectors. Important thing here is do not apply voltage through pin 9 of the DB-9 connector and through the communication/power header at the same time.

\section{e) Power supply:}

The power supply to the PC104 mother board and LCD is given through output of the Switch Mode Power Supply. The SMPS output voltage is $+5 \mathrm{~V},+12 \mathrm{~V}$ DC voltage and main power supply is given to the SMPS as input. These board and LCD works at low DC voltage instead of regular ac power supply.

\section{B. Software Requirements:}

To do this work required software is Qt. The present work is done in Qt which supports PC104 board. Qt is a crossplatform, graphical application development toolkit that enables us to compile and run applications on windows, Mac OSx, Linux and different brands of unix. All tools we need to develop applications are open sourced. It offers APIs for many popular desktop and embedded devices to desktop applications like printers, mobile phones, coffee machines and so on.

\section{IMPLEMENTATION}

a) Interfacing $\mathrm{LCD}$ to $\mathrm{SBC}$ :

In the process of interfacing LCD to SBC, first connect LCD to SBC physically with the help of RS232 cable by identifying corresponding ports. Give power supply to LCD either through board or directly to LCD from SMPS with voltage value of $+5 \mathrm{~V}$. If power supply is giving from SMPS it will be given to power header from pins 1 (Vcc) and 4 (GND). If the LCD shows pre-loaded text on display then ok, otherwise check for connections. After power supply, text is showing on LCD means LCD is working properly otherwise it is not working properly. After interfacing LCD to SBC set basic values to LCD like brightness, contrast, backlight on-time, baud rate etc.

b) Flow chart for writing code:

The above flow chart explains the steps of writing source code using the software Qt. the computer language used for writing the source code is $\mathrm{C}++$ language. This code is written to develop the GUI.

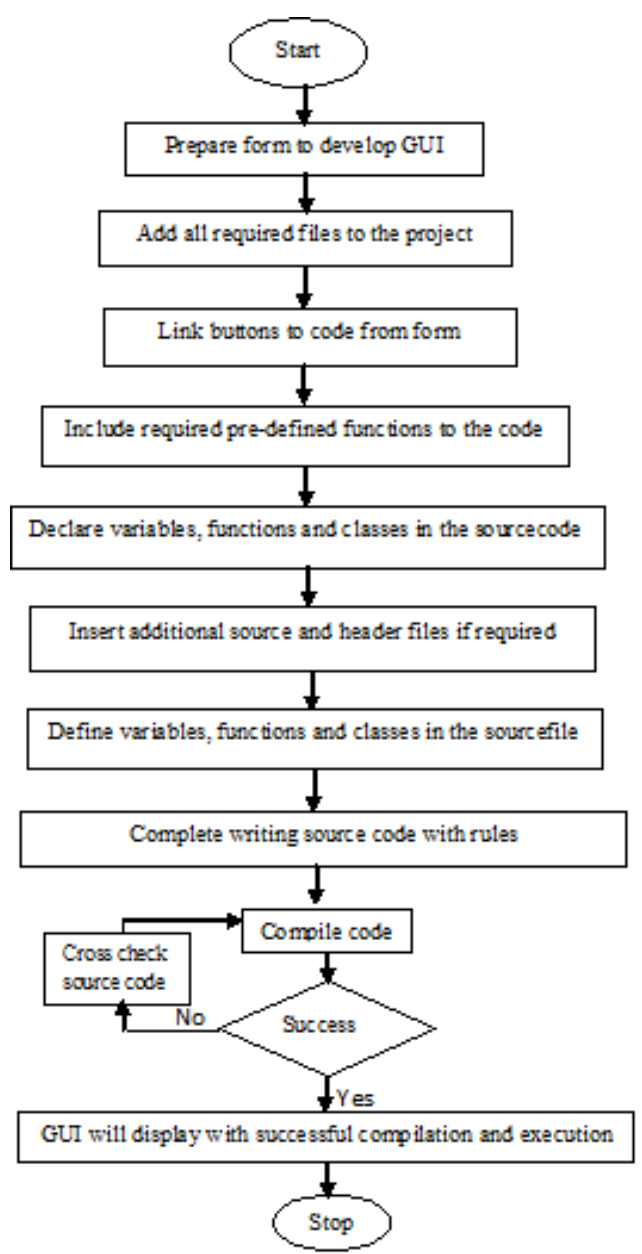

c) Block diagram explanation

The figure shown below represents the block diagram of Interfacing and controlling of LCD with PC104 Single Board Computer. This block diagram consists of LCD, KIB380 expansion module, PC104 mother board, SMPS module, compact flash Type I/II card. Coming to the explanation of the block diagram main power supply of $230 \mathrm{~V}, 50 \mathrm{Hzs}$ is given to the SMPS module with the help of power cable. SMPS will give us is connected to the LCD and both the output DC voltage values are connected to the PC104 mother board.

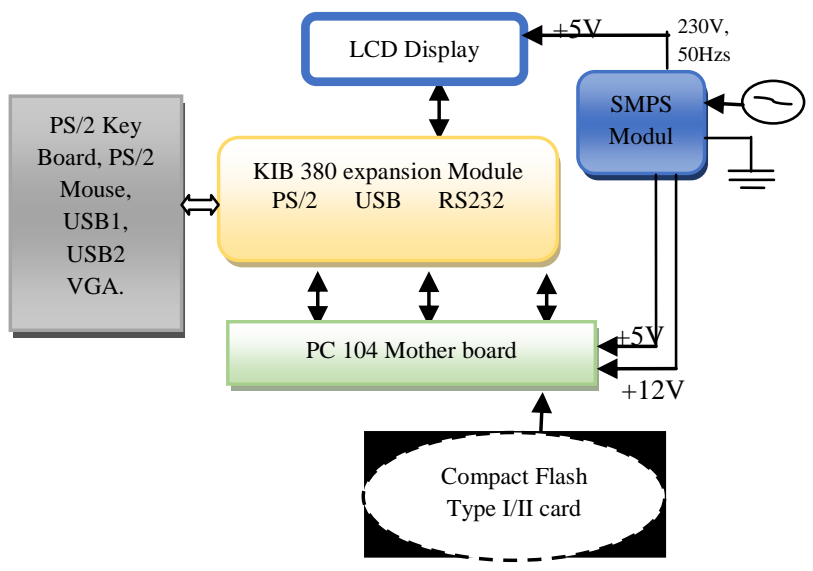

Figure1: Block diagram 
All communication processes, instructions, commands given by user are followed by PC104 mother board. On top side of the PC104 board we have jacks which will allow connecting ribbon cables.

Using these ribbon cables are somewhat difficult compared to normal ports. For that reason again one more KIB380 expansion module is connected to the mother board. We can connect external devices having USB ports, COM ports, and Ethernet ports to the expansion module. LCD is connected to KIB380 expansion module with the help of DB9 connector. Commands to this LCD will be given by user with external devices and these commands will process by $\mathrm{PC} 104$ board, then finally given LCD to respond. In this diagram we can observe one more component called compact flash Type I/II card. It will be inserted at the bottom side of PC104 board at specified socket. The memory size of this card is 4GB and will work same as Hard Disk Drive in our normal personal computer. The required files can be stored in this card by USB devices.

\section{d) Hardware testing method:}

In this work main job is to test the working of LCD. In order to work this LCD, first testing should be done o this LCD. There are two testing methods are available.

\section{Displaying a pre-loaded logo on startup.}

To check whether LCD is working properly or not, LCD manufacturer's will provide their logo at startup screen, means when power supply is given to LCD we should be able to see their logo without fail, otherwise we can come to conclusion that LCD is not working properly. There is one more option that, it is possible to re-write the preloaded logo according to user wish. When power supply is given user defined logo will display at the start-up time.

2. Faulty display detection using full pixel illumination.

There is one more method to test the working of LCD. It is by checking each and every pixel of LCD. To do this work in the developed GUI with the help of source code, one option is there, that is screen check. If we click on that button each and every pixel, character by character will highlight with brightness. If any pixel will not work it will not highlight. Then we can know that there is some problem.

\section{TESTING OF INTERFACE THROUGH GUI}

Source code is written to develop the GUI. GUI developed by writing source code will be as shown below.

If we observe the above GUI, it has so many pushbuttons, line-edits and text-box. By clicking on the pushbutton the corresponding action will takes place based on the type of function.

In the process of developing GUI 3 windows are stacked. These are called as stacked widgets.

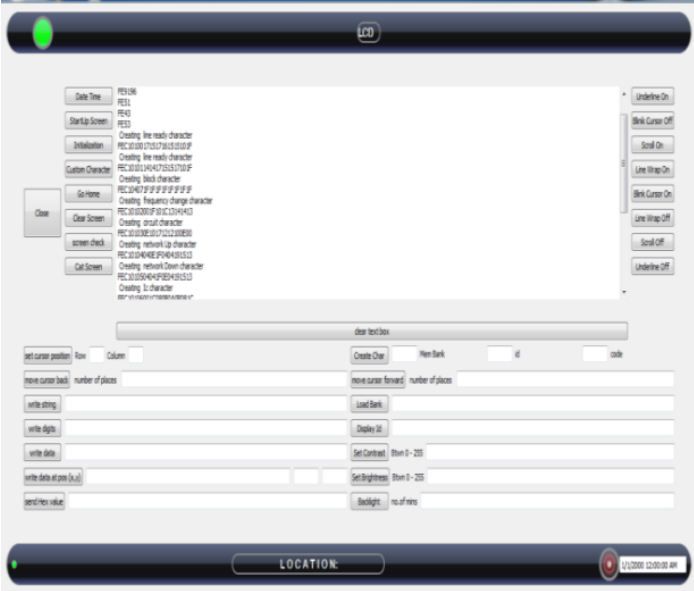

Figure2: First page of GUI

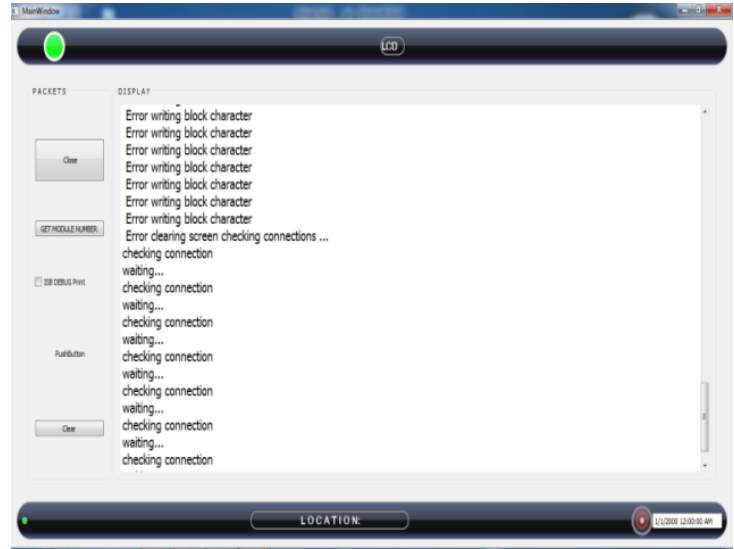

Figure3: Second page of GUI

These will work same as going back and front in web pages. This is used to get module number, Interface Switching Board debug connection.

In this window we have back, front, refresh, stop and go buttons and one line-edit to write the IP address. After entering the link and clicking on GO button, we will get the corresponding link.

\section{SUMMARY, CONCLUSION AND FUTURE SCOPE}

The main purpose of this work was to interface and test LCD with PC104 mother board. GUI was developed using software Qt. But this GUI will show on computer monitor connected to PC104 mother board. From GUI on monitor we will send commands to LCD. This GUI was developed for performing various operations of the communication access terminal such as status display, radio circuit number display, selected frequency value display, characters and numbers send by user etc. Linux operating system was flashed onto compact flash Type I/II card by interfacing the PC104 mother board to a computer. The GUI developed was copied as an executable file onto the flash card for accessing the features of the communication access terminal. The LCD is tested as a final step by using the screen check button through the GUI. 
This work was done for developing the communication access terminal as a single device for accessing voice and data from other communication access terminals which are connected to this. After complete development it contains keypad for performing GUI operations which are explained earlier. Up to this part LCD interfacing and testing was done. By the reference of this work this PC104 mother board has option of cascading of such boards up to 4 . If we cascade these boards communication speed will increase up to 8 times compared to communication speed with single board. Storage capacity also increases.

\section{REFERENCES}

[1] LK162-12/VK162-12, Including the LK136-12-USB and VK162-12 USB Technical Manual, Revision 4.2.

[2] http:// www.fastwel.com.

[3] CPC304 user manual.

[4] The waite groups object-oriented programming in Turbo C++ by Robert Lafore.

[5] C++ GUI programming with Qt3 BRUCE PERENS OPEN SOURCE SERIES by Jasmin Blanchette, Mark summer field.

[6] C++ Common knowledge Essential Intermediate programming by Stephen C. Dewhurst.

[7] Foundation of Qt Development, Build sophisticated graphical applications using one of the world's most powerful multiplatform toolkits, by Johan Thellin.

[8] The C++ programming language, Third Edition by Bjarne stroustrup, copyright @ 1997 by AT \&T, published by Addison Wesley Longman, Inc. ISBN 0-201-88954. All rights reserved.

[9] HLX800_hardware guide.pdf.

[10] 40456A_geode_lx_etx_rdk_pb.pdf

[11]http://www.moxa.com. 